\title{
Analysis of Adjacency Matrix and Neighborhood Associated with Zero Divisor Graph of Finite Commutative Rings
}

\author{
Pranjali Sharma \\ Department of Mathematics, \\ National Institute of Technology, \\ Hamirpur, india
}

\author{
Amit Sharma \\ Department of Mathematics, \\ National Institute of Technology, \\ Hamirpur, india
}

\author{
R.K.Vats \\ Department of Mathematics, \\ National Institute of Technology, \\ Hamirpur, india
}

\begin{abstract}
The present investigation is concerned with zero divisor graph of direct Product of finite commutative rings and to give some new ideas about its corresponding adjacency matrix. In the first section of the paper, we study about neighborhood set of the zero divisor graph of direct product over finite commutative rings. In the second section we discussed some examples of these ring. Finally, some surprsing results (regarding to the adjacency matrix) and theorems also estabilised. The study on neighborhood may be used to represent graph in computer algorthim, neighborhood are used to determine the clustering cofficient of graph and adjacency martix is useful in computer application.
\end{abstract}

Keywords: Zero-divisor, Adjacency Matrix, Commutative ring, Neighborhood, Undirected graph.

\section{AMS Classification: 13Axx; 05C50; 05Cxx; 14B20}

\section{INTRODUCTION}

A graph is an abstract representation of a set of objects where some pairs of the objects are connected by links, here our links is zero divisor. The interconnected objects are represented by mathematical abstractions called vertices, and the links that connect some pairs of vertices are called edges.

The notation of the zero divisor graph $\Gamma R$ of commutative ring was firstly introduced by I. Beck [2], where his motive was coloring of the graph. It had been extended to commutative semi group by De Meyer [4] and for non commutative rings by Redmond (2002). The zero divisor graph also studied by Anderson \& Livingston [3]. They found for a Commutative rings $\Gamma R$ is always connected. At present considerable work has been done in this direction for both commutative $\&$ non commutative rings.

In this article $\mathrm{R}$ denotes finite commutative ring of direct product such as $R=R_{1} \times R_{2}$

Where $R_{1}, R_{2}$ are integral domain however $\mathrm{R}$ is not, i.e., $R_{1}, R_{2}$ both does not have zero divisor but $R$ have. Let $Z[R]$ be set of zero divisor of ring. Let $G^{*}$ be graph of zero divisor of ring. For distinct $a, b \in Z[R]$, $a$ and $b$ are said to be adjacent iff $a \cdot b=0=$ b.a [3]

In the first instance of this paper we construct zero divisor graphs for cartesian product of commutative rings and then we obtain neighborhood set and adjacency matrices corresponding to these graph after that we determine some results of the adjacency matrices. Finally we have proved some theorem on neighborhood of the zero divisor graph of direct product over finite commutative rings as well as for adjacency matrix. For a set A of vertices, the neighborhood of $\mathrm{A}$ is the union of the neighborhoods of the vertices, and so it is the set of all vertices adjacent to at least one member of A. The neighborhood is often denoted $N_{G}(v)$. The open neighborhood of $\mathrm{v}$ is the neighborhood which does not included $\mathrm{v}$. the neighborhood in which $v$ itself is included, called the closed neighborhood and denoted by $N_{G}[v]$. Throughout the paper $\Delta(G)$ denotes the maximum degree of graph. To avoid trivial case, when $G$ has no edges we assume when necessary $\mathrm{R}$ is not integral domain.

In order to illustrate some theorems we analysis the following finite commutative rings such as:

$$
\begin{array}{ll}
\text { a) } & Z_{p} \times Z_{p} \\
\text { b) } & Z_{p}[i] \times Z_{p}[i] \\
& \text { Where } \mathrm{p} \text { is prime number }
\end{array}
$$

We study the basic definition from the graph theory as needed. Basic reference for graph theory [5, 7, 9], for commutative ring theory [2, 10, 11] and for matrix theory [8]. The adjacency matrices corresponding to zero divisor graph is defined as $\mathrm{A}=$ $\left[\mathrm{a}_{\mathrm{i} . \mathrm{j}}\right]$, where

$$
\mathrm{a}_{\mathrm{i} \mathrm{j}}= \begin{cases}1, & \text { If } \mathrm{v}_{\mathrm{i}} \& \mathrm{v}_{\mathrm{j}} \text { represent zero-divisor, i.e., } v_{i} \cdot v_{j}=0 \\ 0, & \text { Otherwise }\end{cases}
$$

, where $\mathrm{v}_{\mathrm{i}}$ and $\mathrm{v}_{\mathrm{j}}$ are vertex of graph.

Let us start the observation for each of them:

$$
\text { a) The Ring } \mathbf{R}=Z_{p} \times Z_{p}
$$

Case a.1: When $\mathbf{p}=\mathbf{2}$, i.e., $\mathbf{R}=Z_{2} \times Z_{2}$

The ring $\mathrm{Z}_{2} \times \mathrm{Z}_{2}$ will be Boolean, individual $\mathrm{Z}_{2}$ has no zero divisor but $Z_{2} \times Z_{2}$ has two zero divisor. The set $Z(R)=\{(1,0),(0,1)\}$ and possible edges will be $\{(1,0),(0,1)\}$ and the graph is given as: 


\section{$(1,0) \bullet \longrightarrow(0,1)$}

Fig.1: Zero divisor graph of $Z_{2} \times Z_{2}$

Neighborhood set $N_{G}[1,0]=\{(1,0),(0,1)\}$,

$N_{G}[0,1]=\{(1,0),(0,1)\}$

$\therefore \underset{v \in G}{\cup} N<v>=\{(1,0),(0,1)\}$ and maximum degree of graph $\Delta(G)=1$.

The matrix for the zero divisor graph of $Z_{2} \times Z_{2}$ is

$$
\mathbf{M}_{1}=\left[\begin{array}{ll}
0 & 1 \\
1 & 0
\end{array}\right]_{2 \times 2}
$$

\section{Properties of adjacency matrix $M_{1}$ :}

I. The determinate of adjacency matrix corresponding to zero divisor graph of $Z_{2} \times Z_{2}$ is -1 .

II. Trace of the adjacency matrix for the zero divisor graph of $Z_{2} \times Z_{2}$ is 0 .

III. Rank of adjacency matrix for the zero divisor graph of $Z_{2} \times Z_{2}$ is 2 .

IV. Eigen values are 1 and -1 , which are distinct, so matrix is diagonalizable.

V. Matrix $\mathrm{M}_{1}$ is Symmetric matrix.

VI. Surprising result is that in the case of Boolean ring the matrix is Involuntary.

Case a. 2: When $\mathbf{p}=\mathbf{3}$, i.e., $\mathbf{R}=Z_{3} \times Z_{3}$

The set of zero divisor is $\mathrm{Z}(\mathrm{R})=\{(0,1),(0,2),(1,0),(2,0)$,$\} and$ the possible edges are $\{(1,0),(0,2)\},,\{(2,0),(0,2)\},\{(0,1),(2,0)\}$, $\{(0,1),(1,0)\}$ and the graph for $\mathrm{Z}(\mathrm{R})$ is

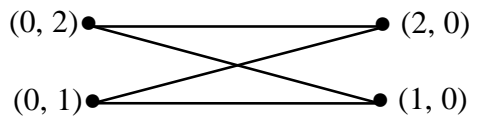

Fig.2: Zero divisor graph of $Z_{3} \times Z_{3}$

Neighborhood set $N_{G}[1,0]=\{(0,1),(0,2),(1,0)\}$,

$N_{G}[0,1]=\{(2,0),(1,0),(0,1)\}$,

$N_{G}[2,0]=\{(0,1),(0,2),(2,0)\}$,

$N_{G}[0,2]=\{(1,0),(0,2),(2,0)\}$

$\therefore \underset{v \in G}{\cup} N<v>=\{(1,0),(0,1),(0,2),(2,0)\}$ and maximum degree of graph $\Delta(G)=2$.

The adjacency matrix for above graph is given as:

$$
\mathbf{M}_{2}=\left[\begin{array}{llll}
0 & 1 & 0 & 1 \\
1 & 0 & 1 & 0 \\
0 & 1 & 0 & 1 \\
1 & 0 & 1 & 0
\end{array}\right]_{4 \times 4}
$$

\section{Properties of adjacency matrix $\mathrm{M}_{2}$ :}

I. The determinate of adjacency matrix corresponding to zero divisor graph of $Z_{3} \times Z_{3}$ is 0 .

II. Trace of the adjacency matrix for the zero divisor graph of $Z_{3} \times Z_{3}$ is 0 .

III. Rank of adjacency matrix for the zero divisor graph of $Z_{3} \times Z_{3}$ is 2 .

IV. Eigen values are $0,0,2$ and -2 and matrix is diagonalizable.

V. Matrix $\mathrm{M}_{2}$ is Symmetric matrix.

Case a.3: When $\mathbf{p}=\mathbf{5}$, i.e., $\mathbf{R}=Z_{5} \times Z_{5}$

The set of the zero divisor of $Z_{5} \times Z_{5}=\{(0,1),(0,2),(1,0),(2$, $0),(3,0),(0,3),(0,4),(4,0)\}$ and the possible edges are $\{(1,0),(0,1)\},\{(1,0),(0,2)\},\{(1,0),(0,3)\},\{(1,0),(0,4)\},\{(2,0)$, $(0,1)\},\{(2,0),(0,2)\},\{(2,0),(0,3)\},\{(2,0),(0,4)\},\{(3,0),(0,1)\}$, $\{(3,0),(0,2)\}, \quad\{(3,0),(0,3)\} \quad\{(3,0),(0,4)\}, \quad\{(4,0),(0,1)\}$, $\{(4,0),(0,2)\},\{(4,0),(0,3)\},\{(4,0),(0,4)\}$ and the graph for $\mathrm{Z}(\mathrm{R})$ is

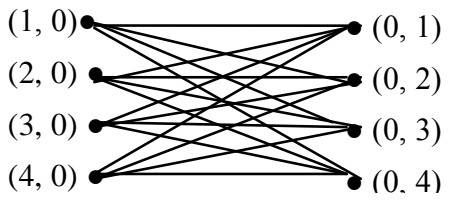

Fig.3: Zero divisor graph of $Z_{5} \times Z_{5}$

Proceeding in similar manner the neighborhood set is as follows $\therefore \underset{v \in G}{\cup} N<v>=\{(0,1),(0,2),(1,0),(2,0),(3,0),(0,3),(0$, $4),(4,0)\}$ and maximum degree of graph $\Delta(G)=4$.

The adjacency matrix corresponding to zero divisor graph is:

$$
\mathbf{M}_{3}=\left[\begin{array}{llllllll}
0 & 1 & 0 & 1 & 0 & 1 & 0 & 1 \\
1 & 0 & 1 & 0 & 1 & 0 & 1 & 0 \\
0 & 1 & 0 & 1 & 0 & 1 & 0 & 1 \\
1 & 0 & 1 & 0 & 1 & 0 & 1 & 0 \\
0 & 1 & 0 & 1 & 0 & 1 & 0 & 1 \\
1 & 0 & 1 & 0 & 1 & 0 & 1 & 0 \\
0 & 1 & 0 & 1 & 0 & 1 & 0 & 1 \\
1 & 0 & 1 & 0 & 1 & 0 & 1 & 0
\end{array}\right]_{8 \times 8}
$$

\section{Properties of adjacency matrix $\mathbf{M}_{3}$ :}

I. The determinate of adjacency matrix corresponding to zero divisor graph of $Z_{5} \times Z_{5}$ is 0 .

II. Trace of the adjacency matrix for the zero divisor graph of $Z_{5} \times Z_{5}$ is 0 .

III. Rank of adjacency matrix for the zero divisor graph of $Z_{5} \times Z_{5}$ is 2 . 
IV. Matrix is diagonalizable.

V. Matrix $\mathrm{M}_{3}$ is Symmetric matrix.

\section{Generalization of the results from analysis over the ring $Z_{p} \times Z_{p}$ : \\ i. Here we conclude that if we take ring $Z_{p} \times Z_{p}, p \neq 2$, then adjacency matrix is always singular. \\ ii. Number of zero divisor in $Z_{p} \times Z_{p}, p \neq 2$, are $2(p-1)$, therefore order of such adjacency matrix is $2(\mathrm{p}-1) \times 2(\mathrm{p}-$ $1)$. \\ iii. The eigen value of adjacency matrix with respect to zero divisor graph of $Z_{p} \times Z_{p}$, is always (p-1) and zero. \\ iv. Rank of the obtained matrix is always 2 . \\ v. The obtained adjacency matrix is always symmetric matrix.}

Theorem a.1: Let $R$ be finite commutative ring of cartesian product and $Z(R)$ be set of zero divisor of ring and let $G$ be zero divisor graph then set of zero divisor is a neighborhood set $N_{G}[v]$ and neighborhood number $n(G)$ is equal to the number of zero divisor of $R$.

Proof: Let $\mathrm{R}$ be finite commutative ring and $\mathrm{Z}(\mathrm{R})$ be set of zero divisor of ring $R$. Let $G$ be zero divisor graph now the set of vertices is said to be neighborhood set if $\underset{v \in G}{\cup} N\langle v\rangle$, where $N\left(v_{i}\right)$ is said to neighborhood of vertex $\mathrm{v}_{\mathrm{i}}$, here $N\left[v_{i}\right]$ is closed neighborhood and then union of neighborhood of all vertices is neighborhood set, Since zero divisor graph is always connected [1], Hence all the zero divisor will come in neighborhood set, So $\mathrm{N}(\mathrm{G})$ is just set of zero divisor \& neighborhood number is cardinality of $\mathrm{N}(\mathrm{G})$, therefore we conclude that number of zero divisor in the set $R$ is equal to neighborhood number $n(G)$.

Theorem a.2: Let $R$ is finite commutative ring of direct product, i.e., $R=Z_{p} \times Z_{p}$. Let $Z(R)$ be set of zero divisor of ring $R$ and $G$ be zero divisor graph then $n(G) \leq 2 \Delta(G)$, where $n(G)$ is neighborhood number and $\Delta(G)$ is the maximum degree of graph.

Proof: Let $\mathrm{R}$ be finite commutative ring and $\mathrm{Z}(\mathrm{R})$ be set of zero divisor $\mathrm{G}$ be zero divisor graph, $\Delta(G)$ denotes maximum degree of graph [12]. It is obtained then $\mathrm{G}$ is always connected [3], i.e., there exist and edge between a pair of vertices. By observing graph, we found that $(\mathrm{a}, 0)$ will connect to $(0, \mathrm{~b}),(0, \mathrm{c}),(0, \mathrm{~d}) \ldots \ldots$, i.e., $\left(a_{i}, 0\right)$ will be related to $\left(0, b_{i}\right) \forall a_{i} \in R_{1}, b_{i} \in R_{2}$. This implies $(a, 0)$ vertex will be connected at least half of zero divisor \& $\Delta(G)$ is maximum degree of vertex so $\Delta(G) \geq \frac{Z(R)}{2}$, equality occurs when $\mathrm{R}=Z_{p} \times Z_{p}$ and $\mathrm{Z}(\mathrm{R})$ is neighborhood set [a.1] and it is shown that $n(G)$ is just equal to number of zero divisor.

Therefore $\Delta(G) \geq \frac{n(G)}{2} \Rightarrow n(G) \leq 2 \Delta(G)$
Theorem a.3: : Let $\mathrm{R}$ be finite commutative ring of Cartesian product, i.e., $R=R_{1} \times R_{2}$, where each $\mathrm{R}_{1}, \mathrm{R}_{2}$ are integral domain $\left(\mathrm{Z}_{\mathrm{p}}\right)$ and let $\mathrm{A}$ be adjacency matrix of corresponding to zero divisor graph then trace of matrix is always zero.

Proof: Let $\left[a_{i, j}\right]_{n \times n}$ be adjacency matrix corresponding to zero divisor graph of finite commutative ring $Z_{p} \times Z_{p}$ as we know that trace $A=\sum_{i=j} a_{i, j}$. In adjacency matrix diagonal entry will be non zero due to self loop., i.e., $v_{i .} v_{j}=0$ then $a_{i, j}, i=1,2,3, \ldots \ldots$ will be non zero. Since above discussed examples shows that zero divisor graph of ring $Z_{p} \times Z_{p}$, no vertex have self loop. Therefore all the diagonal entries are zero. $\therefore \operatorname{tr}(\mathrm{A})=0$.

\section{b) The Ring $\mathbf{R}=Z_{p}[i] \times Z_{p}[i]$,}

Case b.1: When p = 2, i.e., $\mathbf{R}=Z_{2}[i] \times Z_{2}[i]$

In the ring $Z_{2}[i] \times Z_{2}[i]$, the set of zero divisor $Z(R)=$ $\{(1,0),(0,1),(0, i),(i, 0),(0,1+i),(1+i, 0),(1+i, 1+i),(1+i, 1)$, $(1,1+i),(i, 1+i),(1+i, i)\}$ and possible edges will be $\{(1,0),(0$, $1)\},\{(i, 0),(0, i)\},\{(1+i, 0),(0,1+i)\},\{(1,0),(0, i)\},\{(1,0),(0$, $1+\mathrm{i})\},\{(\mathrm{i}, 0),(0,1)\},\{(\mathrm{i}, 0),(0,1+\mathrm{i})\},\{(1+\mathrm{i}, 0),(0,1)\},\{(1+\mathrm{i}$, $0),(0, \mathrm{i})\},\{(1+\mathrm{i}, 0),(1+\mathrm{i}, 1+\mathrm{i})\},\{(0,1+\mathrm{i}),(1+\mathrm{i}, 1+\mathrm{i})\},\{(1+\mathrm{i}$, $0),(1+\mathrm{i}, 0)\},\{(0,1+\mathrm{i}),(0,1+\mathrm{i})\},\{(1+\mathrm{i}, 0),(1+\mathrm{i}, 1)\},,\{(0,1+\mathrm{i}),(1$, $1+i)\},\{(1+i, 1+i),(1+i, 1+i)\},\{(1+i, 0),(1+i, i)\},\{(0,1+i),(i$, $1+\mathrm{i})\},\{(0,1+\mathrm{i}),(0,1+\mathrm{i})\},\{(1+\mathrm{i}, 0),(1+\mathrm{i}, 0)\}$ and the graph is given as:

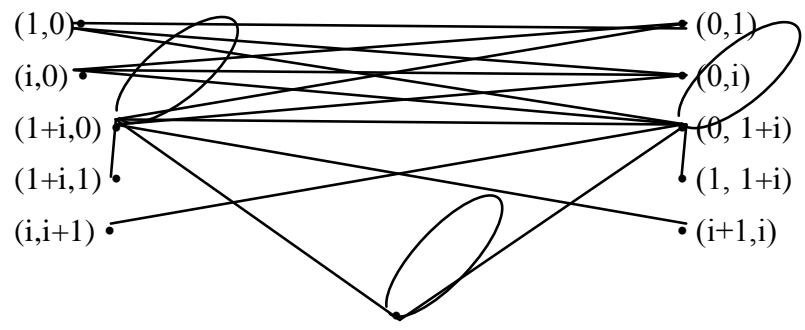

$(1+\mathrm{i}, 1+\mathrm{i})$

Fig.4: Zero divisor graph of $Z_{2}[i] \times Z_{2}[i]$

We can also determine the neighborhood set from the above graph in similar manner

$\therefore \underset{v \in G}{\cup} N<v>=\{(1,0),(0,1),(0, \mathrm{i}),(\mathrm{i}, 0),(0,1+\mathrm{i}),(1+\mathrm{i}, 0)$, $(1+i, 1+i),(1+i, 1),(1,1+i),(i, 1+i),(1+i, i)\}$ and maximum degree of graph $\Delta(G)=8$.

The adjacency matrix for the above graph is as follows: 


$$
\mathbf{M}_{4}=\left[\begin{array}{lllllllllll}
0 & 1 & 0 & 1 & 0 & 1 & 0 & 0 & 0 & 0 & 0 \\
1 & 0 & 1 & 0 & 1 & 0 & 0 & 0 & 0 & 0 & 0 \\
0 & 1 & 0 & 1 & 0 & 1 & 0 & 0 & 0 & 0 & 0 \\
1 & 0 & 1 & 0 & 1 & 0 & 0 & 0 & 0 & 0 & 0 \\
0 & 1 & 0 & 1 & 1 & 1 & 1 & 0 & 1 & 0 & 1 \\
1 & 0 & 1 & 0 & 1 & 1 & 0 & 1 & 1 & 1 & 0 \\
0 & 0 & 0 & 0 & 1 & 0 & 0 & 0 & 0 & 0 & 0 \\
0 & 0 & 0 & 0 & 0 & 1 & 0 & 0 & 0 & 0 & 0 \\
0 & 0 & 0 & 0 & 1 & 1 & 0 & 0 & 1 & 0 & 0 \\
0 & 0 & 0 & 0 & 0 & 1 & 0 & 0 & 0 & 0 & 0 \\
0 & 0 & 0 & 0 & 1 & 0 & 0 & 0 & 0 & 0 & 0
\end{array}\right]_{11 \times 11}
$$

\section{Properties of adjacency matrix $\mathbf{M}_{4}$ :}

I. The determinate of adjacency matrix corresponding to zero divisor graph of $Z_{2}[i] \times Z_{2}[i]$ is 0 .

II. Trace of the adjacency matrix for the zero divisor graph of $Z_{2}[i] \times Z_{2}[i]$ is 3 .

III. Rank of adjacency matrix for the zero divisor graph of $Z_{2}[i] \times Z_{2}[i]$ is 7 .

IV. Matrix $\mathrm{M}_{4}$ is Symmetric matrix.

\section{Case b.2: When $\mathbf{p}=\mathbf{3}$, i.e., $\mathbf{R}=Z_{3}[i] \times Z_{3}[i]$}

In the ring, $Z_{3}[i] \times Z_{3}[i]$ the set of zero divisor $Z(R)=\{(1,0)$, $(0,1),(0,2),(2,0),(0, i),(i, 0),(0,2 i),(2 i, 0),(0,1+i),(1+i, 0)$, $(0,1+2 \mathrm{i}),(1+2 \mathrm{i}, 0),(0,2+\mathrm{i}),(2+\mathrm{i}, 0),(0,2+2 \mathrm{i}),(2+2 \mathrm{i}, 0)\}$ and possible edges will be $\{(1,0),(0,1)\},\{(0,1),(2,0)\},\{(0,1),(\mathrm{i}, 0)\}$, $\{(0,1),(2 \mathrm{i}, 0)\},\{(0,1),(1+\mathrm{i}, 0)\},\{(0,1),(1+2 \mathrm{i}, 0)\},\{(0,1),(2+\mathrm{i}, 0)\}$, $\{(0,1),(2+2 \mathrm{i}, 0)\}, \quad\{(0,2),(1,0)\}, \quad\{(0,2),(2,0)\}, \quad\{(0,2),(\mathrm{i}, 0)\}$, $\{(0,2),(2 \mathrm{i}, 0)\},\{(0,2),(1+\mathrm{i}, 0)\},\{(0,2),(1+2 \mathrm{i}, 0)\},\{(0,2),(2+\mathrm{i}, 0)\}$, $\{(0,2),(2+2 \mathrm{i}, 0)\}, \quad\{(0, \mathrm{i}),(1,0)\}, \quad\{(0, \mathrm{i}),(2,0)\}, \quad\{(0, \mathrm{i}),(\mathrm{i}, 0)\}$, $\{(0, \mathrm{i}),(2 \mathrm{i}, 0)\},\{(0, \mathrm{i}),(1+\mathrm{i}, 0)\},\{(0, \mathrm{i}),(1+2 \mathrm{i}, 0)\},\{(0, \mathrm{i}),(2+\mathrm{i}, 0)\}$, $\{(0, \mathrm{i}),(2+2 \mathrm{i}, 0)\},\{(0,1+\mathrm{i}),(1,0)\},\{(0,1+\mathrm{i}),(2,0)\},\{(0,1+\mathrm{i}),(\mathrm{i}, 0)\}$, $\{(0,1+\mathrm{i}),(2 \mathrm{i}, 0)\}, \quad\{(0,1+\mathrm{i}),(1+\mathrm{i}, 0)\}, \quad\{(0,1+\mathrm{i}),(1+2 \mathrm{i}, 0)\}, \quad\{(0$, $1+\mathrm{i}),(2+\mathrm{i}, 0)\},\{(0,1+\mathrm{i}),(2+2 \mathrm{i}, 0)\},\{(0,1+2 \mathrm{i}),(1,0)\},\{(0,1+2 \mathrm{i}),(2$, $0)\}, \quad\{(0,1+2 \mathrm{i}),(\mathrm{i}, 0)\}, \quad\{(0,1+2 \mathrm{i}),(2 \mathrm{i}, 0)\}, \quad\{(0,1+2 \mathrm{i}),(1+\mathrm{i}, 0)\}$, $\{(0,1+2 \mathrm{i}),(1+2 \mathrm{i}, 0)\},\{(0,1+2 \mathrm{i}),(2+\mathrm{i}, 0)\}, \quad\{(0,1+2 \mathrm{i}),(2+2 \mathrm{i}, 0)\}$, $\{(0,2+\mathrm{i}),(1,0)\},\{(0,2+\mathrm{i}),(2,0)\},\{(0,2+\mathrm{i}),(\mathrm{i}, 0)\},\{(0,2+\mathrm{i}),(2 \mathrm{i}, 0)\}$, $\{(0,2+\mathrm{i}),(1+\mathrm{i}, 0)\}, \quad\{(0,2+\quad \mathrm{i}),(1+2 \mathrm{i}, 0)\}, \quad\{(0,2+\mathrm{i}),(2+\mathrm{i}, 0)\}$, $\{(0,2+\mathrm{i}),(2+2 \mathrm{i}, 0)\}, \quad\{(0,2+2 \mathrm{i}),(1,0)\}, \quad\{(0, \quad 2+2 \mathrm{i}),(2,0)\}$, $\{(0,2+2 \mathrm{i}),(\mathrm{i}, 0)\}, \quad\{(0,2+2 \mathrm{i}),(2 \mathrm{i}, 0)\}, \quad\{(0,2+2 \mathrm{i}),(1+\mathrm{i}, 0)\}$, $\{(0,2+2 \mathrm{i}),(1+2 \mathrm{i}, 0)\},\{(0,2+2 \mathrm{i}),(2+\mathrm{i}, 0)\},\{(0,2+2 \mathrm{i}),(2+2 \mathrm{i}, 0)\}$. and the graph is given as:

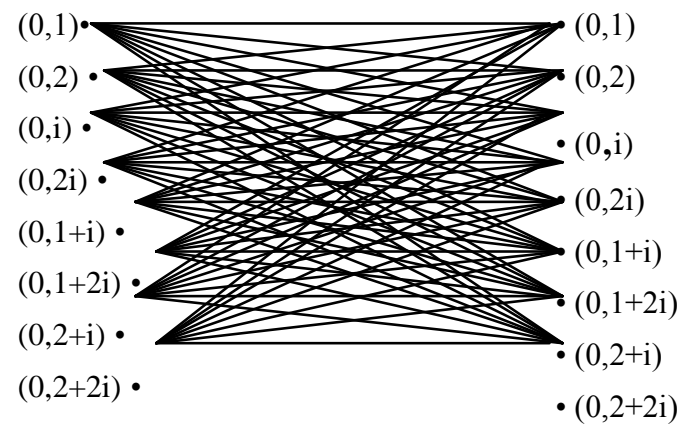

Fig.5: Zero divisor graph of $Z_{3}[i] \times Z_{3}[i]$
We also can determine the neighborhood set from the above graph in similar manner

$\therefore \underset{v \in G}{\cup} N<v>=\{(1,0),(0,1),(0,2),(2,0),(0, \mathrm{i}),(\mathrm{i}, 0),(0,2 \mathrm{i})$, $(2 \mathrm{i}, 0),(0,1+\mathrm{i}),(1+\mathrm{i}, 0),(0,1+2 \mathrm{i}),(1+2 \mathrm{i}, 0),(0,2+\mathrm{i}),(2+\mathrm{i}, 0)$, $(0,2+2 \mathrm{i}),(2+2 \mathrm{i}, 0)\}$ and maximum degree of graph $\Delta(G)=8$.

The adjacency matrix for the above graph is as follows:

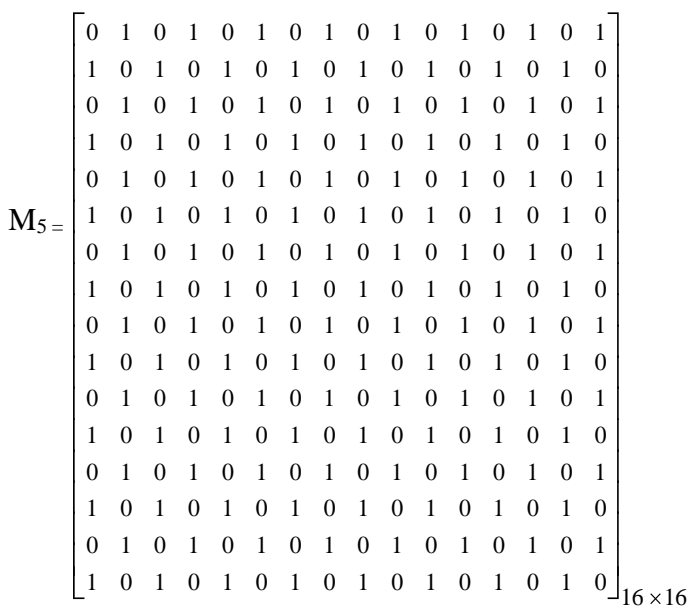

\section{Properties of adjacency matrix $\mathrm{M}_{\mathbf{5}}$ :}

I. The determinate of adjacency matrix corresponding to zero divisor graph of $Z_{3}[i] \times Z_{3}[i]$ is 0 .

II. Trace of the adjacency matrix for the zero divisor graph of $Z_{3}[i] \times Z_{3}[i]$ is 0 .

III. Rank of adjacency matrix for the zero divisor graph of $Z_{3}[i] \times Z_{3}[i]$ is 2 .

IV. Matrix $\mathrm{M}_{5}$ is Symmetric matrix.

Theorem b.2.1: Let $R$ be finite commutative ring of Cartesian product of Gaussian integer under modulo $p$ i.e., $R=Z_{p}[i] \times Z_{p}[i]$. Let $A=\left[\begin{array}{ll}a_{i} & j\end{array}\right]$ be adjacency matrix corresponding to zero divisor graph of $Z_{p}[i] \times Z_{p}[i]$, then trace of adjacency matrix is always a natural matrix, $n>1$.

Proof: Let $\left[a_{i j}\right]_{n \times n}$ be adjacency matrix corresponding to zero divisor graph $\mathrm{G}$ of finite commutative ring $Z_{p}[i] \times Z_{p}[i] \&$ as we know that trace $A=\sum_{i=j} a_{i j}$. In adjacency matrix diagonal entry will be non-zero only due to self loop., i.e., $v_{i .} v_{i}=0$ then $a_{i}$, $\mathrm{i}=1,2,3, \ldots$ will be non-zero at least for one $\mathrm{i}$. . Here we observe that in $Z_{p}[i] \times Z_{p}[i]$ zero divisors graph must contain a self loop. Therefore the diagonal entries of adjacency matrix $\left[a_{i j}\right]_{n \times n}$ corresponding to zero divisor graph of finite commutative ring of direct product, i.e., $Z_{p}[i] \times Z_{p}[i]$ are non- 
zero for any at least one i. Hence trace $A=\sum_{i=j} a_{i, j}$ is always a natural number $\mathrm{n}, \mathrm{n}>1$.

Theorem b.2.2: Let $R$ be finite commutative ring and $Z(R)$ be set of zero divisor of ring and let $G$ be zero divisor graph of finite commutative ring of direct product except Boolean ring,i.e., $R \neq Z_{2} \times Z_{2}$. Let $A=\left[a_{i} j_{j}\right]$. be adjacency matrix corresponding to zero divisor graph. The adjacency matrix with respect to $G$ is always singular.

Proof: Let $\mathrm{R}$ be finite commutative ring and $\mathrm{Z}(\mathrm{R})$ be set of zero divisor of ring $R$. Let $G$ be zero divisor graph from discussed examples, we analysis that each $\left(\mathrm{a}_{\mathrm{i}}, 0\right)$ is related to $\left(0, \mathrm{~b}_{\mathrm{i}}\right) \forall$ $a_{i} \in R_{1}, b_{i} \in R_{2}$. Then in adjacency matrix corresponding to zero divisor graph of commutative ring of direct product at least two vectors will be linearly dependent. Therefore rank of matrix is less than the order of matrix. Hence determinant of matrix is zero.

Theorem $\boldsymbol{b}$.2.3: Let $R$ be finite commutative ring of Cartesian product. Let $G$ denotes the zero divisor graph of ring. Let $n(G)$ )

and $n(G)$ be neighborhood number of zero divisor graph of ring

$R$ and its component graph respectively. Then $n(G)=n(\bar{G})$.

Proof: Let $\mathrm{R}$ be finite commutative ring and $\mathrm{Z}(\mathrm{R})$ be set of zero divisor of ring $\mathrm{R}$. Let $\mathrm{G}$ be zero divisor graph as it shown that set of zero divisor is neighborhood set [a.1] and $n(G)$ equal the number of the zero divisors. The complement of the zero divisor graph two vertices are joined iff $a . b \neq 0, a \neq 0$ and $b \neq 0$. From $\bar{G}$, we observed that each closed neighborhood of vertices contains at least two vertices then $\underset{v \in Z(R)}{\cup} N<v>$ will again contain all the zero divisors. Thus in this way the neighborhood number $n(\bar{G})$ is also equal to $n(G)$, i.e., $n(G)=n(\bar{G})$.

\section{CONCLUSION:}

In this paper, we study adjacency matrices for zero divisor graph over finite commutative rings of direct product. Adjacency array representation can be generalized to store additional information.
Graphs are the most ubiquitous models of both natural and human made structures. In computer science, zero divisor graphs are used to represent networks of communication, network flow, clique problems. Art gallery and museum guard problem are a well-studied visibility problem in computational geometry. Neighborhood can be used to represent graphs in computer algorithms, through adjacency matrix representation. It is also used to determine the clustering coefficient of the graph, which is measure of the average density of its neighborhood.

\section{REFERENCES}

[1] Gallian J. A., Abstract Algebra, Narosa Publishing House, ISBN: 81-7319-269-3(1998).

[2] Beck I., “Coloring of Commutating Ring”, J. Algebra 116, 208-226(1988).

[3] Anderson D.F., P.S. Livingston, "The Zero-divisor Graph of Commutative Ring”, Journal of Algebra 217, 434-447(1999).

[4] DeMeyer F.R., T.Mckenzie, K.Schneider, "The Zero-divisor Graph of a Commutative Semi-groups", Semi Group Forum 65, 206-214(2002)

[5] Diestel R., Graph Theory, Springer-Verlag, Newyork, 1977.

[6] Harary F., Graph Theory, Addison-Wesley, Reading, MA, 1972.

[7] Kaplansky I., Commutative Rings, Univ. of Chicago Press, Chicago, 1974.

[8] Bhat V.K, Ravi Raina, "A Note on Zero-divisor Graph over Rings”, Int. J. Contemp. Math. Sci. 2(14), 667-671(2007).

[9] Bollabs B., Graph Theory-An Introductory Course, SpringerVerlag, Newyork, 1979.

[10] Atiyah M.F, I.G, Macdonald, "Introduction to Commutative Algebra”, Addison-Wesley, Reading, MA, 1989.

[11] Nafiz Abu Jaradeh Emad Abu Osba and Salah Ai-Addasi, "Zero Divisor graph for the ring of Gaussian integers modulo n", Taylor \& Francis, Communications in algebra, 36: 38653877(2008).

[12] Kulli V.R., S.C. Sigarkanti, "Further result on the neighborhood number of a graph" Indian J. Pure 